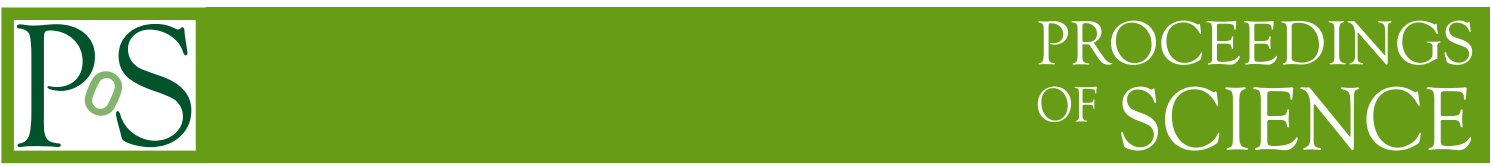

\title{
Perspectives in Neutrino and Multi Messenger Physics
}

\section{Soo-Bong Kim* \\ Seoul National University, Republic of Korea}

E-mail: sbkesnu.ac.kr

The impressive progress in neutrino physics has opened a new field of neutrino science such as neutrino astronomy or neutrino geoscience. The measurement of all three neutrino mixing angles has established a comprehensive picture of neutrino transformation and needs extremely accurate measurements of the mixing angles for a unitarity test. A rather large value of the smallest mixing angle $\theta_{13}$ makes it possible to determine the $\mathrm{CP}$ violating phase and the neutrino mass ordering without a neutrino factory. A next round of neutrino experiments are under consideration or preparation to make a future neutrino science. The Hyper-Kamiokande experiment belongs to one of those efforts and will use a 250 kton water Cherenkov detector together with a neutrino beam produced by the J-PARC. A second Hyper-Kamiokande detector in South Korea, called Korean Neutrino Observatory (KNO), is proposed to enhance physics sensitivities based on a larger overburden of the detector and a longer baseline $(\sim 1100 \mathrm{~km})$ of the accelerator neutrino beam.. In this talk we present an overview of the $\mathrm{KNO}$ and describe possible physics and astronomy potentials.

XVII International Workshop on Neutrino Telescopes

13-17 March 2017

Venezia, Italy

\footnotetext{
*Speaker.
} 


\section{Introduction}

There has been a great progress in understanding the neutrino sector of elementary particle physics in the last two decades. The discovery of neutrino oscillation [1] unambiguously tells existence of neutrino mass and is a direct indication of physics beyond the Standard Model. It provides a unique, new window to explore at the Grand Unification energy scale. The definitive measurement of the smallest neutrino mixing angle $\theta_{13}$ [2] has firmly established the framework of neutrino oscillation among three flavors. The neutrino oscillation is described by a unitary Pontecorvo-Maki-Nakagawa-Sakata matrix with three mixing angles $\left(\theta_{12}, \theta_{23}\right.$ and $\left.\theta_{13}\right)$ and one CP phase angle [3]. A rather large value of $\theta_{13}$ has motivated a next round of neutrino experiments under consideration or preparation to determine the $\mathrm{CP}$ violating phase and neutrino mass ordering without a neutrino factory.

Due to the impressive progress in the neutrino physics, a new field of "neutrino science" has begun. It includes neutrino particle physics, neutrino astronomy, neutrino cosmology and neutrino application. In the next section an overview of the neutrino science is presented, and then the KNO project is introduced and discussed.

\section{Neutrino science}

\subsection{Neutrino particle physics}

Neutrino is not an unknown particle any more, but still a mysterious particle. There are remaining fundamental questions on neutrino to be answered yet. We do not know its absolute mass while the squared-mass-differences are measured. The neutrino mass ordering is not known, either. We do not know whether it is a Dirac or Majorana particle. In the recent development there is an exciting hint on a possibly large value of the leptonic CP violating phase close to $3 \pi / 2$ [4]. Existence of a sterile neutrino is another intriguing question of particle physics. Various experiments are on-going, under construction or under consideration to solve those fundamental problems using accelerator, reactor, cosmic and radioactive source neutrinos.

\subsection{Neutrino astronomy}

R. Davis and M. Koshiba received the 2002 Nobel physics prize for their pioneering contributions to astrophysics, in particular for detection of cosmic neutrinos. They opened a new field of neutrino astronomy by detecting neutrinos from the center of the Sun and a supernova explosion. The IceCube detector at the South Pole recently observed high-energy neutrinos from the Universe. Neutrino has demonstrated to be a good tool to probe the interior of a star. The next observation of a supernova neutrino burst will reveal the detailed mechanism of supernova explosion and cooling. An early neutrino sighting of a supernova implosion will provide a valuable information for a next historical observation by an optical telescope.

\subsection{Neutrino cosmology}

Cosmological neutrinos play an important role in the evolution and structure formation of the Universe, modifying some of the cosmological observables. The precision of present cosmological data can be used to learn about neutrino properties. In particular, cosmology associated with 
the cosmic microwave background provides information on the absolute scale of neutrino masses, complementary to beta decay and neutrinoless double-beta decay experiments. There are good perspectives from future cosmological measurements which are expected to be sensitive to the minimum values of neutrino masses.

\subsection{Neutrino application}

The electron density profile of the Earth can be reconstructed from the energy spectrum of neutrinos that traverse the Earth medium and go through the matter oscillation. The inner Earth chemical composition is not well understood [5] while its matter density is reasonably well known by seismic measurements [6]. A future neutrino detector will be sensitive to make an experimental confirmation on an iron core of the Earth based on neutrino oscillations in matter. It will likely open a new field of neutrino geophysics. It is under discussion and development that neutrino detectors could help detect nuclear weapons. Physicists also have proposed a futuristic but not necessarily impossible technology that would use an ultra-high energy neutrino beam to destroy nuclear weapons [7]. However, they stressed that the method is well beyond the capabilities of current particle accelerators and would require substantial R\&D and financial investment.

\section{Second Hyper-Kamiokande in Korea}

A large value of $\theta_{13}$ has motivated several future neutrino experiments under construction or preparation to determine the $\mathrm{CP}$ violating phase or/and the neutrino mass ordering. They are DUNE, Hyper-Kamiokande (HK), JUNO, REO-50, PINGU, ORCA, INO, and so on. The current HK strategy is staged construction of two 250 kton detectors near the Super-Kamiokande site, 295 $\mathrm{km}$ away and $2.5^{\circ}$ off-axis (OA) from the J-PARC neutrino beam. The HK experiment employs a ring-imaging water Cherenkov detector technique to detect neutrino interactions and a possible proton decay. It is a cylindrical tank with a diameter of $74 \mathrm{~m}$ and height of $60 \mathrm{~m}$ The detector will be located in the Tochibora mine and under Mt. Nijuugo, with an overburden of $650 \mathrm{~m}$ of rock. An overview of the HK cavern and detector design R\&D, upgraded neutrino beam and near detector, and expected physics sensitivities can be found in the HK design report [8]. A schematic view of the first HK detector is shown in Fig. 1. While the first HK iproject is under financial request, the international collaboration has recommended to consider the second HK in South Korea [9].

\subsection{Overview of Korean Neutrino Observatory (KNO)}

An alternative configuration of locating the second HK detector in Korea at a baseline of $\sim 1100 \mathrm{~km}$ is seriously recommended to enhance the sensitivity for the leptonic CP violation and the neutrino mass ordering. Studies show that a larger matter effect and the second oscillation maximum occurrence with a detector in Korea can improve the sensitivity in comparison to two HK detectors at Kamioka and allow breaking the degeneracy of oscillation parameters. The KNO also provides an enhanced physics program on neutrinos from the Sun, supernova and dark matter, and geophysics using atmospheric neutrinos because of benefits from the larger overburden in the Korean site.

There were earlier ideas on a large water Cherenkov detector in Korea using a J-PARC neutrino beam [10]. Several authors have proposed a two baseline experiment with a second detector in 


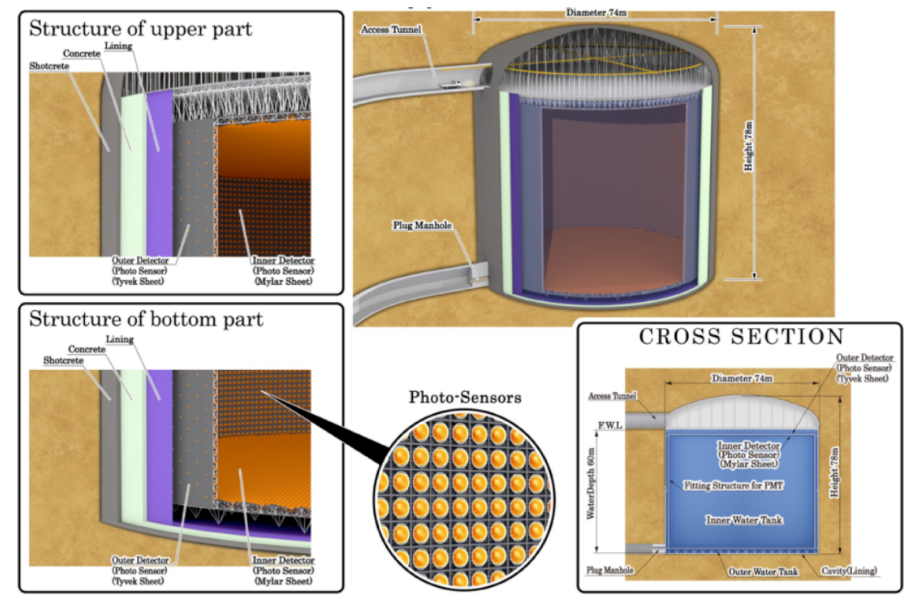

Figure 1: Schematic view of the first HK detector.

Korea for improved measurements on the CP violation and neutrino mass ordering [11, 12]. Three international workshops were held in Korea and Japan in 2005, 2006 and 2007 [13]. The smallest mixing angle $\theta_{13}$ value was not known yet, and therefore the detector size and mass could not be determined at the time. Now more realistic studies and a detector design are possible due to the precisely measured value of $\theta_{13}$ [2].

The axis of the J-PARC neutrino beam emerges upwards out of the sea between Japan and Korea. The southern part of the Korean peninsula is exposed to he 1-3 degree OA neutrino beams with baselines of 1000-1300 km as shown in Fig. 2. There are enough candidate sites of mountains over $1000 \mathrm{~m}$ height within the range. This allows for the optimization of the OA angle based on physics sensitivities and systematic error consideration.

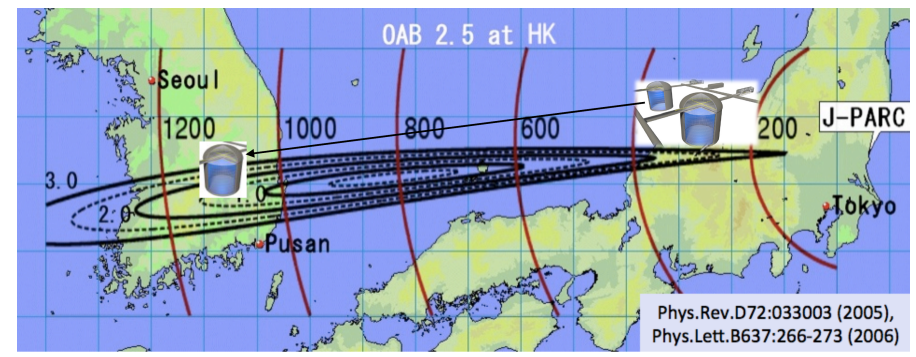

Figure 2: Contour map of the J-PARC off-axis beam to Korea [12]

The second oscillation maximum takes place near $E_{v}=0.6 \mathrm{GeV}$ at the $\sim 1100 \mathrm{~km}$ baseline while the first oscillation maximum occurs at the energy at Kamioka.. The clear separation among different CP phases and between mass orderings is observed at the second oscillation maximum. The KNO can be the most sensitive to the CP phase determination if it is placed at 2.5 degrees of OA angle, the same as HK in Kamioka. The J-PARC neutrino beam spectrum at the OA angle peaks at $E_{v}=0.6 \mathrm{GeV}$ with a narrow energy band as shown in Fig. 3. Having identical OA angles of the J-PARC beam for for HK and KNO. a ratio measurement between HK and KNO would 
greatly reduce the uncertainties of the neutrino beam flux and spectrum. On the other hand, the maximum sensitivity for determining the neutrino mass ordering is possible by a neutrino beam of $E_{V}>1 \mathrm{GeV}$ where the first oscillation maximum takes place. The J-PARC neutrino beam spectrum peaks above $1 \mathrm{GeV}$ with a wider energy band when its $\mathrm{OA}$ angle is less than 1.5 degrees. In this case, the neutrino flux becomes less in the energy region near the second-oscillation maximum, but still remains enough for the satisfactory $\mathrm{CP}$-phase sensitivity.
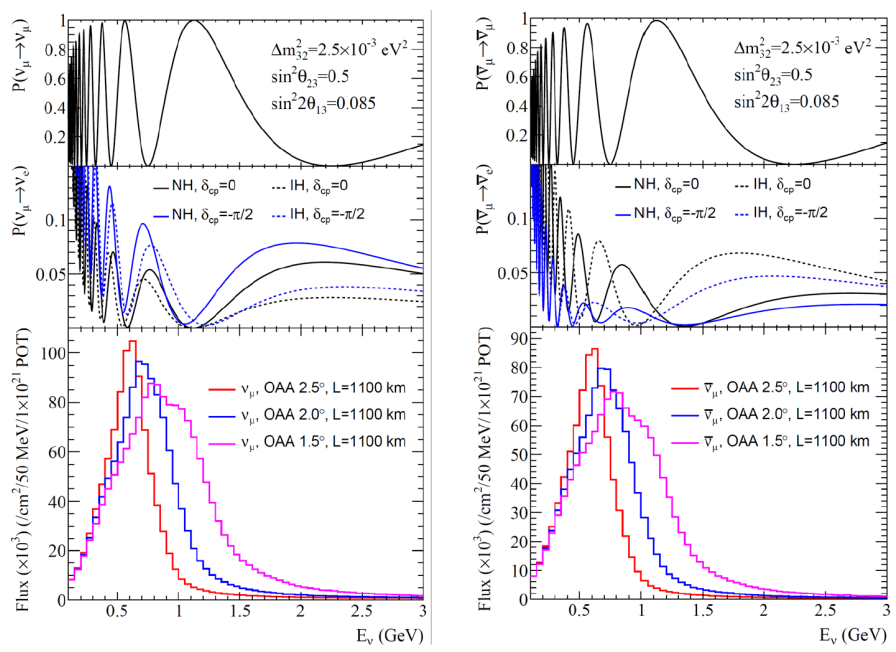

Figure 3: Expected J-PARC neutrino (left) and antineutrino (right) spectra and oscillation probabilities at KNO assuming the baseline of $1100 \mathrm{~km}$. Several OA angles are considered for comparison. The second oscillation maximum takes place at $\sim 0.6 \mathrm{GeV}$ at $\mathrm{KNO}$ while the first one is at $\sim 1.5 \mathrm{GeV}$

The Korean rocks are in general made of granite, hard enough to build a large cavern. A search for mountains higher than $1000 \mathrm{~m}$ has been made to find several candidate sites. Two candidate sites are selected among those as shown in Fig. 4: Mt. Bisul at 1.3 degrees of OA angle and Mt. Bohyun at 2.2 degrees. The KNO in the Mt. Bisul would provide a high sensitivity for the neutrino mass ordering determination due to a large matter effect as well as an improved sensitivity for the $\mathrm{CP}$ phase measurement because of both first and second oscillation maxima. A geological survey has been made to obtain a rock hardness profile. Both candidate sites are based on rocks of classes 1 or 2 , excellent for constructing a large cavern.

\subsection{Enhanced neutrino physics potentials}

The tiny neutrino mass and the large neutrino mixing compared to quarks indicate that the origin of neutrino mass is from physics beyond the Standard Model, e.g., the seesaw mechanism. Test of the unitarity of three generation neutrino mixing paradigm and precise determination of the mixing parameters would provide an effective tool for constraining new physics as demonstrated in quark mixing. For example, precision measurement of $\mathrm{CP}$ violating phase could distinguish different types of flavor symmetries in Grand Unification Theory.

The complementary information from the second detector in Korea at different baseline length could extend the sensitivity of the overall HK measurements significantly. Due to a factor of three 


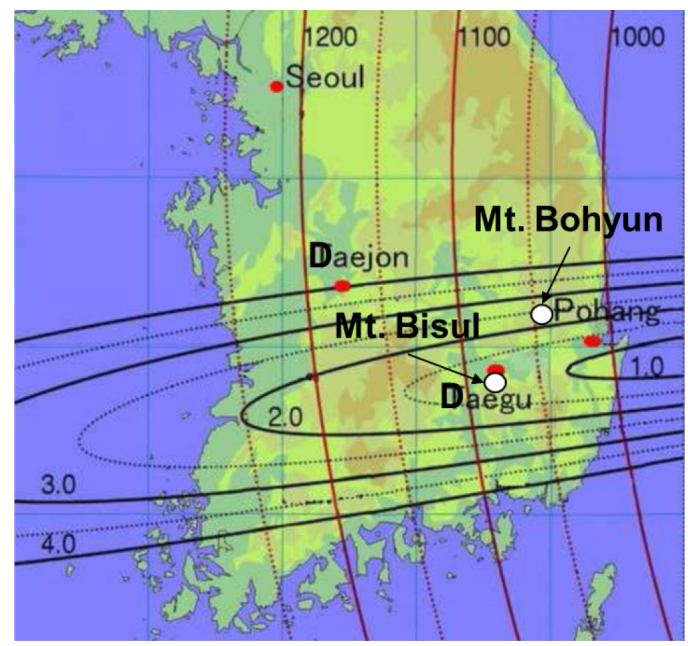

Figure 4: Two candidate sites for the second HK detector in Korea. Mt. Bisul is located near the city of Daegu and at 1.3 degrees of OA angle, and Mt. Bohyun at Youngcheon and at 2.2 degrees of OA angle. Mt. Bisul is $1084 \mathrm{~m}$ high and provides excellent accessibility. Mt. Bohyun is $1124 \mathrm{~m}$ high and accommodates a national optical telescope on the top.

enhancement of $\mathrm{CP}$ violation effect at the second oscillation maximum, the systematic uncertainties at KNO are reduced accordingly. The two different baseline oscillation measurement allows breaking the degeneracy of oscillation parameters and constraints the physics beyond the PMNS paradigm. Matter effect creates a fake $\mathrm{CP}$ violation result introducing difference between neutrino and antineutrino oscillations. Non-standard neutrino interaction may also cause a similar fake CP effect. Both of these fake effects can be resolved by the two baseline experimental data.

The sensitivity enhancement of a second detector comes from the second oscillation maximum which generates three times larger $\mathrm{CP}$ violation effect than that of the first oscillation maximum. The KNO at Mt. Bisul makes the best constraint on the $\mathrm{CP}$ violating phase near the maximal value of $\mathrm{CP}$ violation. Significant improvement in the precision of $\mathrm{CP}$ violating phase measurement is obtained as shown in Fig. 5.
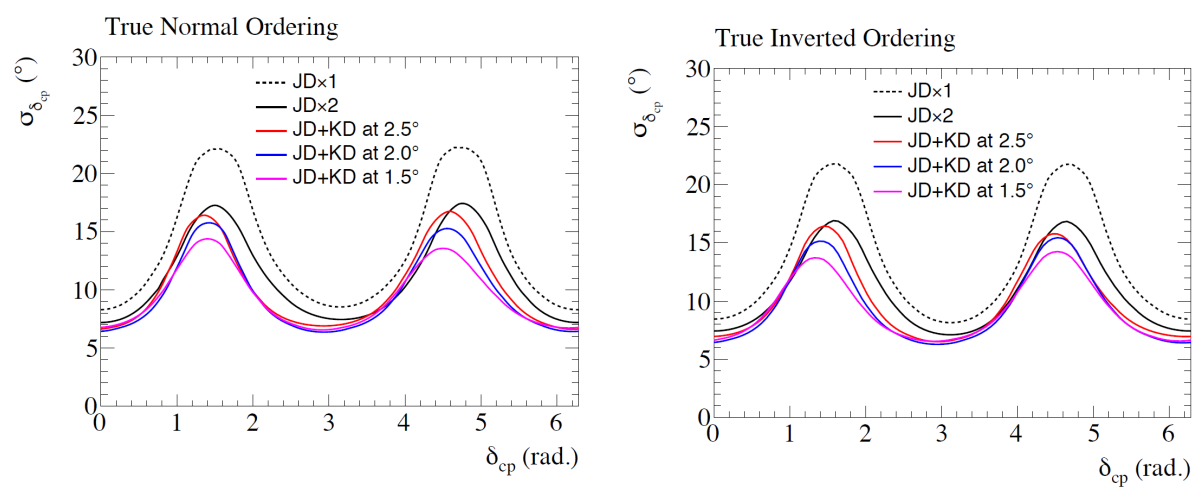

Figure 5: Precision of the $\mathrm{CP}$ violating phase measurement as a function of the true phase value. No prior knowledge of the neutrino mass ordering is assumed. 
Neutrinos propagating in matter experience interactions with matter. The matter effect modifies the neutrino oscillation probability. The KNO with a longer baseline is more sensitive to determine the neutrino mass ordering because of increasing matter effect. For a baseline of $\sim 1100$ $\mathrm{km}$ the separation between two mass ordering cases becomes stronger at the first oscillation maximum, and thus the neutrino beam with a smaller OA angle enhances the sensitivity of neutrino mass ordering determination. The significance to reject the wrong mass ordering is shown in Fig. 6. It is the largest for the configuration with the $\mathrm{KNO}$ at $1.5^{\circ} \mathrm{OA}$ angle because the more on-axis position gives more events in the $1-2 \mathrm{GeV}$ range of larger matter effect.
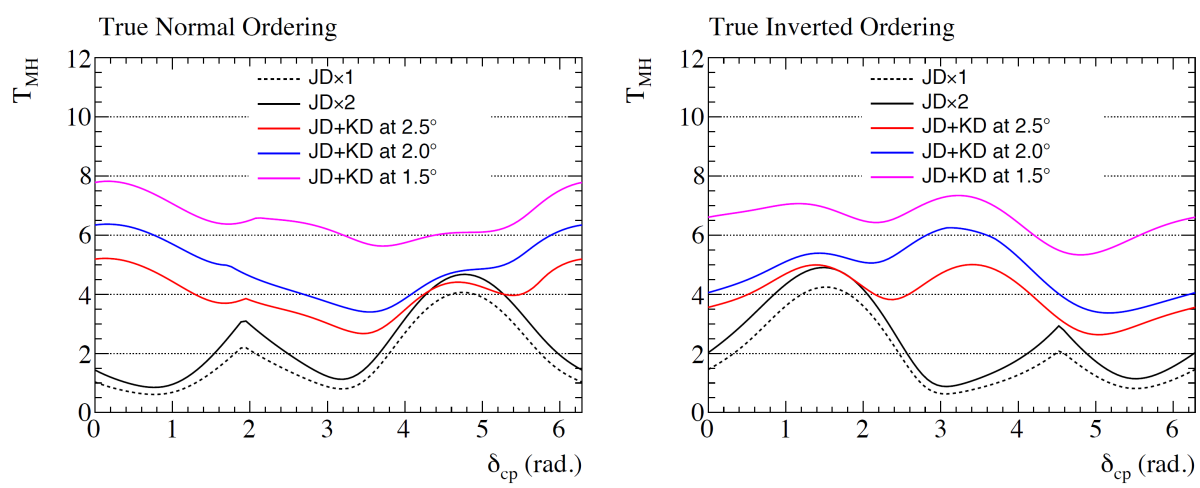

Figure 6: Significance for rejecting the wrong mass ordering as a function of the true value of $\mathrm{CP}$ violating phase and the true mass ordering.

The mass ordering sensitivity can be further enhanced by combining the atmospheric neutrino data as shown in Fig. 7. Note that the enhancement depends on the value and uncertainty of $\theta_{23}$. It is clear that the sensitivity is also enhanced by the KNO as the second HK detector in Korea.
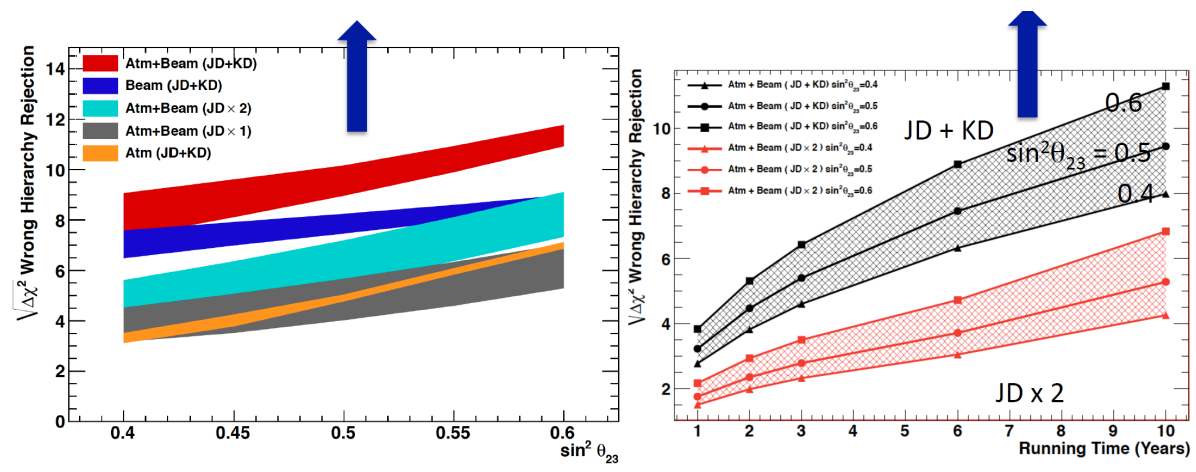

Figure 7: Significance for rejecting the wrong mass ordering as a function of the true value of $\theta_{23}$ (left) and as a function of the running time (right).

The KNO using the J-PARC neutrino beam can be a powerful tool for probing non-standard physics related with neutrinos, including (1) quantum decoherence, (2) tiny violation of Lorentz symmetry with or without CPT invariance, and (3) non-standard neutrino interactions with matter. The KNO is expected to make significant improvements of the bounds on possible new physics 
effects in the neutrino sector. The improvement is possible because the spectral distortion of the neutrino beam can be measured more accurately using two detector configuration and larger matter effect with a longer baseline.

\subsection{Enhanced neutrino astronomy potentials}

In addition to the long baseline program with multiple detectors at different baselines, there are additional benefits in the non-accelerator program by placing the second detector in Korea. The prospective deeper detector site with an overburden of $\sim 1000 \mathrm{~m}$ will result in reduction of cosmic muon flux and spallation isotope production in turn. The next advantage comes from the geographical separation between two detectors.

A lower spallation background level would introduce better sensitivity for solar neutrino measurements: (1) the day and night asymmetry due to the MSW matter effect in the Earth, (2) detection of solar hep neutrinos, (3) monitoring of short time variation of the temperature in the solar core, and (4) upturn of the energy spectrum of solar neutrino due to the neutrino oscillations in vacuum and in matter.

A lower spallation background rate will enhance detection sensitivity of the supernova relic neutrinos (SRN) below $20 \mathrm{MeV}$ and thus improve the study of supernova bursts in the past. The lower background rate will also enhance the sensitivity of detecting a neutrino burst from a supernova explosion.

HK is likely the first experiment to confirm an Earth iron core at $3 \sigma$ confidence level. The observation relies on precise measurement of atmospheric muon neutrino disappearance and electron neutrino appearance in the energy range of $1-8 \mathrm{GeV}$ as a function of the zenith angle. The KNO detector will bring the benefit of having two detectors at different locations which could potentially lead to reduced systematic uncertainties related to the atmospheric neutrino flux.

HK can perform a search for physics beyond the Standard Model in the form of self-annihilating dark matter captured in the Sun, Earth or Galactic halo. The atmospheric neutrino is a main background against a signal from dark matter annihilation in the Sun. The KNO at the second site could reduce systematic uncertainties associated with atmospheric neutrino fluxes.

\section{Summary and conclusion}

An alternative option of locating the KNO as the second HK detector at a baseline of $\sim 1100$ $\mathrm{km}$ is seriously pursued to enhance the sensitivity for the leptonic CP violation and the neutrino mass ordering. A larger matter effect and the second oscillation maximum occurrence with the KNO can improve the sensitivity in comparison to two HK detectors at Kamioka. The KNO also provides an enhanced physics program on neutrinos from the Sun, supernova and dark matter, and geophysics using atmospheric neutrinos because of a lower spallation background rate from the larger overburden in the Korean site. Based on the improvement in various physics sensitivities, the KNO shows a viable and attractive option as an alternative to the default HK plan with two detectors in Kamioka. 


\section{Acknowledgments}

This work is supported by the Samsung Science \& Technology Foundation grant No. SSTFBA1402-06.

\section{References}

[1] Y. Fukuda et al. (Super-Kamiokande Collaboration), Phys. Rev. Lett. 81 (1998) 1562-1567; Q.R. Ahmad et al. (SNO Collaboration), Phys. Rev. Lett. 87 (2001) 071301; Q.R. Ahmad et al. (SNO Collaboration), Phys. Rev. Lett. 89 (2002) 011301.

[2] Y. Abe et al. (Double Chooz Collaboration), Phys. Rev. Lett. 108 (2012) 131801; F.P. An et al. (Daya Bay Collaboration), Phys. Rev. Lett. 108 (2012) 171803; J.K. Ahn et al. (RENO Collaboration), Phys. Rev. Lett. 108 (2012) 191802.

[3] B. Pontecorvo, Sov. Phys. JETP 7 (1958) 172; Z. Maki, M. Nakagawa, and S. Sakata, Prog. Theor. Phys. 28 (1962) 870.

[4] K. Abe et al. (T2K Collaboration), Phys. Rev. Lett. 112 (2014) 061802; J. Bian et al. (NOvA Collaboration), [hep-ex/1510.05708] (2015).

[5] W. McDonough and S. Sun, Chem. Geol. 120 (1995) 223.

[6] A.M. Dziewonski and D.L. Anderson, Phys. Earth Planet. Interiors 25 (1981) 297.

[7] H. Sugawara et al. truction of nuclear bombs using ultrahigh-energy neutrino beam [hep-ph/0305062] (2003).

[8] K. Abe et al. (Hyper-Kamiokande Proto-Collaboration), Hyper-Kamiokande Design Report KEK-preprint-2016-21 (2016).

[9] K. Abe et al. (Hyper-Kamiokande Proto-Collaboration), Physics Potentials with the Second Hyper-Kamiokande Detector in Korea, [hep-ex/1611.06118] (2016).

[10] S.B. Kim, in Proceedings of the KOSEF-JSPS Joint Seminar on New Developments in Neutrino Physics, KIAS at Seoul, Korea (2000) p. 182; K. Hagiwara, Nucl. Phys. Proc. Suppl. 137 (2004) 84.

[11] M. Ishitsuka, T. Kajita, H. Minakata and H. Nunokawa, Phys. Rev. D 72 (2005) 033003; T. Kajita, H. Minakata, S. Nakayama and H. Nunokawa, Phys. Rev. D 75 (2007) 013006; T. Dufour. T. Kajita, E. Kearns and K. Okumura, Phys. Rev. D 81 (2010) 093001.

[12] K. Hagiwara, N. Okamura, and K. Senda, Phys. Lett. B 637 (2006) 266, Erratum: Phys. Lett. B 641 (2006) 491; K. Hagiwara, N. Okamura, and K. Senda, Phys. Rev. D 76 (2007) 093002.

[13] T. Kajita and H. Minakata, Highlights in the T2KK Workshops in 2005 and 2006, http://www-rccn.icrr.u-tokyo.ac.jp/workshop/T2KK07/proceedings/t2kk-pdf/003-014.pdf; T. Kajita, S.B. Kim and A. Rubbia, [hep-ex/0808.0650] (2008); Proceedings edited by T. Kajita and S.B. Kim, 3rd International Workshop on a Far Detector in Korea for the J-PARC Neutrino Beam, 30 Sep. - 1 Oct. 2007, Tokyo, Japan, Tokyo Univ. Acad. Press (2008). 